\title{
Las teorías profesionales y las 5 crisis del periodismo
}

\author{
Eva Aladro Vico* \\ ealadro@ucm.es
}

\begin{abstract}
(Abstracts y palabras clave al final del artículo)
Propuesto: 8 de febrero de 2013

Evaluado: 15 de febrero de 2013

Aceptado: 21 de febrero de 2013
\end{abstract}

El presente artículo tiene dos fines fundamentales: uno es analizar el estado del periodismo profesional de nuestros tiempos, pero no en una panorámica más o menos intuitiva, sino a partir de las perspectivas que las distintas fases de las denominadas Teorias Profesionales del periodismo han ido proporcionando. Se da la significativa coincidencia de que cada período de las teorías profesionales ha puesto el dedo en la llaga en las sucesivas crisis que han conducido al periodismo mundial al actual piélago de dificultades.

En mi opinión, el periodismo ha pasado por cinco fases de crisis sucesivas que han ido asolando su diseño y función profesional y su valor social, hasta llegar a la situación actual de pérdida acelerada de peso en la sociedad del siglo XXI. Las crisis que se han sucedido son:

-moral/ética (años 1920)

-socio-profesional (1960)

-política (1980)

-tecnológica (2000)

-financiera (2010)

Estas cinco fases de crisis se han visto diagnosticadas y acompañadas por investigaciones académicas. Existe una relación entre las teorías académicas de cada período y los fundamentos metodológicos o epistemológicos en que se articulan, y el estado del periodismo. Pretendemos incluso afirmar que existe responsabilidad también entre la investigación académica y los sucesivos problemas de la profesión. El periodismo profesional y los estudios académicos profesionales han mantenido un divor-

* Universidad Complutense de Madrid. Una versión inicial de este texto se ha presentado como ponencia en el Congreso Internacional sobre Periodismo y Nuevas Tecnologías CONNEIC celebrado en Monterrey, México, en marzo de 2013. 
cio y separación creciente de objetivos y de enfoques que es también parte responsable en la situación de crisis total actual.

Pretendemos así analizar incluso nexos de relación que responsabilizan de la situación al mundo teórico académico, que, como el mundo profesional del periodismo, que ha sido demasiado reticente a la aportación comprometida, a la innovación, a la propuesta de soluciones y al conocimiento en profundidad de la situación, por adolecer del mismo sentido de autodefensa profesional que se criticó al mundo del periodismo activo.

La segunda finalidad es, a partir de dicho análisis, plantear que el periodismo profesional de calidad, como el comunismo como sistema social, no es que haya fracasado, sino que no se ha llegado a poner en práctica. Saliendo de la mera anécdota, es posible reflotar conceptos clave y categorías del periodismo anteriores a las mismas Teorías Profesionales, que en su núcleo contienen todavía, en muchos casos de manera inédita, un valor enorme para la reconstitución del periodismo.

Al fondo de estas reflexiones, vemos que la crisis del periodismo precede y acompaña a la crisis sociopolítica y económica del comienzo del siglo XXI, existiendo también no solamente una complementariedad entre ambas, sino una relación causa efecto.

\section{LAS PRIMERAS TEORÍAS PROFESIONALES: LAS TEORÍAS MORALES Y DEMOCRÁTICAS DEL PERIODISMO}

Los estudios y análisis sobre el periodismo profesional siempre nos remiten, como una habitual letanía, a las formulaciones profesionales de los primeros autores de la Escuela de Chicago, periodistas que como Walter Lippmann o Robert Ezra Park, formularon una teoría de la noticia y del periodismo en las que lo esencial era fundamentalmente la línea de valores de una profesión naciente. (vid en este mismo número de CIC los ejemplos de Park (1941, 2013) y de Lippmann (1919, 1920).

Lippmann formuló en A Test of News (1919), y en Liberty for the News (1920) toda una teoría cognitiva sobre el papel de las noticias en la corrección de los fenómenos psicosociales de distorsión generados por la Opinión Pública (McChesney 2011: 153). Aunque se insiste en la profundidad con la que este autor conceptualizó el pseudoentorno de la opinión masiva, y su efectivo papel en las sociedades nacientes del siglo XX, en realidad ni Park ni Lippmann consideraban que opinión pública y noticias fueran necesariamente una pareja bien avenida. Al contrario, las teorías profesionales de los primeros años aún ambicionaban, por la influencia de la filosofía y de teorías sociológicas sólidas, un concepto del periodismo profesional no ligado a su poder factitivo o legitimador de las opiniones masivas o del poder social que controla las mismas.

En $A$ Test of News Lippman articula dos fenómenos bien diferentes, que luego las teorías sociales han llegado a fusionar: el periodismo y la difusión de información, por un lado, y la opinión pública por otro. La articulación de estos dos niveles de acción social es fundamental. Los periodistas tienen la función de generar y controlar la solidez de la opinión pública con la adecuada difusión de información, que 
contrarreste fenómenos de manipulación, propaganda, envenenamiento del ambiente social, corrupción y reaccionarismo (Lippman en Mc Chesney 2011:153, traducción en este mismo número de CIC).

En los años 20, los teóricos distinguen claramente entre información y propaganda. No existe todavía la idea difusa de un mismo fenómeno psicosocial, como un hecho consumado. El "supremo deber del periodismo es proporcionar a la opinión pública el alimento adecuado para que pueda defender la democracia" (2011:154). Las ideas de Lippman y de Merz son asombrosamente innovadoras, a casi un siglo de su publicación. Dicen ambos autores que el periodismo cumple una tarea fundamental en el siglo XX, que es la de liberar a los ciudadanos mediante la información, para que éstos puedan actuar controlando a sus gobiernos, luchando contra la "fabricación del consenso" (la expresión proviene originalmente de este texto de 1920): "Los gobiernos tienden a operar controlando la opinión mediante su acción. El cambio en el lugar de la soberanía, del pueblo al gobierno, ha premiado la fabricación del consenso... sin protección contra la propaganda, sin calidad en las evidencias, sin criterios de atención, la sustancia viva de toda decisión popular se expone a prejuicios y a la explotación infinita de todo tipo" (Mc Chesney 2011:154, vid aquí mismo la traducción en el texto de este autor).

Con lucidez asombrosa, Lippman dice que la presente crisis de la democracia occidental es antes una crisis del periodismo (2011:154): ¿no es increíble que se repita esta misma situación, cien años después?. La acción social, el verdadero funcionamiento asambleario y basado en la deliberación de decisiones a partir de la información de calidad y la formación ciudadana, es vital para la democracia, según el genial autor americano. Y esa acción social genuina que controla la corrupción política está fundada en el deseo de conocer, en la búsqueda de la información, que es uno de los primeros móviles para conseguir la libertad (Mc Chesney 2011:161). Si esa función no se ejerce ni se valora, la democracia degenerará en dictadura de todo signo, porque el autogobierno no se produce (2005:160).

\section{LA PROFESIONALIZACIÓN DEL PERIODISMO Y LA DESPEDIDA DE LA FUNCIÓN AUTOGESTORA DEL PERIODISMO EN LA DEMOCRA- CIA. LAS TEORÍAS SOCIOLÓGICAS PROFESIONALES.}

Debían pasar muchos años hasta que las teorías de la opinión pública de Elizabeth Noelle Neumann ratificaran el matrimonio de conveniencia entre clima de opinión y medios informativos, que definitivamente zanja el debate entre opinión pública y función moral del periodismo. Los primeros tratadistas anteriores a esta fase, empero, señalaban el valor de acicate que las noticias debían tener en un mundo fascinado por la propaganda, la manipulación y los fenómenos de masas. Pero los autores de las primeras Teorías del Periodismo se convirtieron pronto en fuentes para citas - salero que simplemente ornamentaban teorías más pragmáticas y a la vez más escépticas sobre el periodismo profesional.

En la primera mitad del siglo XX brotan las teorías que por primera vez explican el denominado profesionalismo periodístico, es decir, el resultado de la consoli- 
dación del periodismo como actividad económica organizada. Los análisis de Warren Breed (1955) en las redacciones, los estudios de David Manning White (1950) sobre la función censora de los informadores, sus hábitos y dietas informativas, y las primeras formas de distorsión involuntaria debidas a la circunstancia de estabilización laboral del periodismo, coinciden con una cierta pérdida de calidad y de actividad socialmente evolucionaria en las redacciones estadounidenses de los años 40 y 50 .

Al mismo tiempo que el periodismo se aburguesa, las primeras formas de rutina profesional son analizadas por los tratadistas de las nacientes Teorías Profesionales. Se describe la ósmosis profesional y la tendencia endogámica de las prácticas profesionales, y la distancia que ya separa la praxis profesional del ideal al que debe tender. El periodismo empieza a ser una profesión bajo su propio control y al margen de los valores morales. Y al tiempo que se da la despedida al profundo valor fundacional de la democracia y libertad que el periodismo tiene en los teóricos de Chicago, los autores académicos empiezan a dar una imagen efectiva, y no ideal, del periodismo activo ( vid toda la recensión de las teorías en Valbuena 1998).

Puede verse este proceso como un abandono conjunto: los autores comienzan a mostrar la visión crítica y escéptica del periodismo real. Tiene gran peso el alejamiento sociológico, científico, respecto del interés por una función real del periodismo en la vida democrática. Los autores de las teorías profesionales denuncian, pero no llaman al periodismo a cambiar este naciente proceso de profesionalismo por encima de su valor deontológico básico. Los periódicos dejan de pretender ser avanzadillas de la sociedad, luchadores contra el crimen organizado, o fuentes de independencia absoluta para la consecución del progreso urbano. Pero los sociólogos que estudian el periodismo no se interesan por ese factor, pues su función es más científica que política.

El surgimiento de las teorías sobre el profesionalismo y la consolidación del periodismo profesional estabilizado son paralelos. Conforme este periodismo se extiende, arraiga y produce efectos sociales, las teorías van adquiriendo un mayor tinte crítico y escéptico, como ocurre con los análisis de Jeremy Tunstall (1976) sobre los prototipos de informadores, entre los que ya aparece el periodista villano, el antihéroe informativo, como una realidad profesional.

Cuando Jeremy Tunstall establece sus categorías profesionales, el periodismo es una profesión de éxito, socialmente aceptada, con poder político en aumento. El profesionalismo es el culto al ejercicio profesional de un oficio que tiene un poder muy asertivo en la vida social. Muy atrás quedan ya, olvidados en los estantes de las Facultades de Periodismo, los volúmenes de autores que reflexionaron sobre moral y profesión. Simone Weil por ejemplo $(1934,1986)$, reivindicando en la base del ejercicio profesional la superación de la contradicción intrínseca en la moral del trabajo. Weil ahondaba en la paradoja que se produce cuando se ejerce una profesión de manera obligada, para ganarse la vida, y cómo ello cercena la posibilidad de no producir mediante ella un mal a la naturaleza o a la vida. La superación de este dilema moral y el logro de la libertad moral convierte a la profesión y al oficio en un ejercicio metafísico, casi iniciático, como estudiaran autores de la filosofía o de la axiología (de René Guénon a Max Weber). 
El periodismo profesional jamás planteó honduras de esta naturaleza en su concepción del oficio. El profesionalismo, es decir, el culto a la profesión por encima de todo, y a pesar de los dilemas morales, es una de las características esenciales del periodismo. Cuando los teóricos noruegos Galtung y Ruge (1960) analizan los criterios de selección de las noticias internacionales y demuestran que los periodistas se guían por criterios de comodidad laboral tanto como por criterios realmente objetivos, no hacen sino constatar que el profesionalismo, el ejercicio profesional como un valor superior a cualquier criterio moral, ontológico u objetivo, se ha impuesto como una realidad laboral en el periodismo moderno. El periodista y profesor Tom Goldsdtein (1986) formulaba en los años 80 su tesis de que los periodistas pagan cualquier precio por una noticia, ejemplificándolo en diversos casos conocidos de la época. Célebres escándalos periodísticos de los 80 le sirven para ilustrar cómo los periodistas pueden imponer su trabajo a cualquier otra idea, obteniendo las noticias de manera falsaria e inventándolas si es preciso. Pero los años 80 son ya el período de profesionalismo salvaje que antecede a la siguiente fase de crisis en el periodismo profesional, que definitivamente instala en el sector una visión economicista y suicida de la vida laboral.

\section{PROFESIONALISMO SALVAJE PARA UNA PROFESIÓN EXPUESTA AL JUICIO PÚBLICO. LA CONSOLIDACIÓN DE LAS TEORÍAS SOCIOPRO- FESIONALES}

Gaye Tuchman (1983) o Mark Fishman (1985) presentan sus brillantísimos análisis sociológicos de redacciones y medios anglosajones en los años en que el profesionalismo salvaje es ya el teorema de base del ejercicio profesional. El éxito profesional, en los análisis de estos autores y de toda la generación de estudiosos europeos y americanos del mundo productivo de las noticias, domina un oficio en el que los criterios selectivos son arbitrarios o interesados, en el que la noticiabilidad se calcula y programa, la publicación se negocia, la facticidad de la información se apuntala tras poderes fácticos reales, y las noticias son, fundamentalmente, novedades sin cambio (Tuchman 1986).

Los autores que describen con rigor sociológico absoluto el vaciado de valores del periodismo tradicional, y el ejercicio de una profesión sin más ley que la de obtener éxito y evadir las críticas menudean en todos los países europeos y anglosajones. Algunos autores, como Philip Elliott, expertos en Sociología de las Profesiones, señalan y rastrean una condición indispensable para entender la crisis crónica del periodismo profesional: el periodismo es una profesión gremial, formada desde una base humilde, con muy bajo nivel de corporativismo, y sobre todo, es una profesión intelectual expuesta al juicio y crítica públicos sobre sus procedimientos y resultados.

El hecho de carecer de una conformación profesional colegiada y organizada en corporaciones y nichos académicos de mayor arraigo colegial, el hecho de ser una profesión sometida al juicio y evaluación públicos masivos, hace del periodismo una profesión autodefensiva, que proclama constantemente su valor y su posición en la 
vida social, mediante rigideces en las rutinas, agresividad en las actuaciones y un carácter impositivo en su actitud social en general (Elliott 1975)

El profesionalismo salvaje, es decir, la tendencia a ejercer la profesión periodística de manera agresiva, guiada por una falta de articulación de sus motivos de fondo, un interés desmedido por la obtención de beneficio, es en gran medida resultado de la inseguridad profesional del periodismo, una profesión sin aura medicinal, sin conocimiento iniciático, y expuesta a la crítica constante. Los trabajos de Tuchman, Elliott o Fishman muestran cómo los periodistas se aferran a sus rutinas, se apoyan en el poder, y buscan la indiscutibilidad de sus procedimientos, para evadirse de las críticas.

El control de la propia profesión sobre su desarrollo en el siglo XX lleva a abandonar ideas más románticas del periodismo y a acoger masivamente un ideario autodefensivo poco meditado. El paso siguiente, en la siguiente década, será en dos direcciones: evolucionar hacia el sensacionalismo absoluto en la búsqueda del beneficio constante y dejando completamente obsoleta la imagen ideal del periodista, o marchar hacia el periodismo de declaraciones, de referencia y protocolario, alineado con las fuentes de poder e igualmente cerrado a cualquier duda sobre su posición social privilegiada. Ambas maniobras suicidas conducen, al final del siglo XX, a una pérdida absoluta del crédito y confianza social en el medio ciudadano. Bastará con que un leve soplo de cambio tecnológico derribe las raíces podridas de una profesión decidida a no cambiar pese a quien pese.

Es muy importante señalar que parte de la responsabilidad de la decadencia de una profesión de enorme valor social y moral como es el periodismo está en la ciencia profesional desarrollada en la universidad. Aunque las teorías sociológicas detectaron y diagnosticaron a tiempo el progresivo deterioro moral profesional, lo cierto es que su sesgo fue siempre hacia el estudio de ese fenómeno desde un punto de vista de especimen sociológico. Las teorías sociológicas estuvieron más interesadas en la pirueta teórica de mostrar la degeneración profesional del periodismo como algo obvio y hasta natural, que en un análisis de los conceptos altruistas y progresistas que se estaban perdiendo en el mundo profesional. Mientras el periodismo daba una imagen negativa, las teorías se limitaban a registrar fríamente el fenómeno, pero sin intervenir positivamente. La radiografía de una profesión marcada por los sesgos, el desvío y distorsión involuntarios, es simplemente un éxito académico sin consecuencias en la propia profesión. Se hace patente que el periodismo y la universidad están completamente divorciados en sus objetivos y fines.

Como afirmaba recientemente Daniel Santoro (2004), el periodismo llega a fosilizar la cognición social. Pero la sociología profesional sobre el periodismo también termina por aceptar esa fosilización como si se tratara de una cuestión inevitable en el concepto profesional de los periodistas. 


\section{CIENCIA ORGANIZADA Y PERIODISMO DEGRADADO}

Cuando llegamos a los años 90 la teoría profesional periodística adquiere un grado de solidez enorme con la incorporación de metodologías de investigación socio-discursivas, etnometodológicas y sociométricas de precisión.

Una línea de trabajo investigador viene desarrollando desde los años 70 el análisis de la trama de facticidad que los medios generan en alianza con las fuentes de poder (Paletz y Entman 1976). La alianza con las fuentes de poder llega a su momento culminante en el periodismo de referencia de los años 90, en el que se impone la jerarquización y selección absoluta de las fuentes, el periodismo de declaraciones oficiales, la dependencia de fuentes de poder privado y público de todo tipo, y el ejercicio profesional completamente viciado desde su base. Los estudios sobre la llamada Prensa de Referencia Dominante (Imbert y Vidal Beneyto 1996) muestran al desnudo la pérdida de espontaneidad y de carácter en una prensa de calidad completamente al servicio del interés de poder.

Las teorías que estudian la dependencia del poder político y las tramas de alianzas entre periódicos, medios informativos, y políticos en el poder abundan en los años 80 y 90 en el siglo XX. (el Glasgow Media Group, 1976). Estas teorías críticas constatan la influencia profunda que la dependencia del poder tiene en los modos de redactar, de recopilar o de difundir la información. Seguimos encontrándonos ante análisis abiertamente ninguneados o despreciados por el periodismo activo, que no toma nota apenas de todo el derivar profesional hacia la pérdida de valor social de este oficio.

Los estudios de análisis discursivo y socio-semiótico de la última década del siglo XX describen en detalle las maniobras y operaciones léxicas activadoras de una visión profundamente estática y conservadora del estatus quo social, en el que los periódicos poderosos tienen un inmenso influjo (Van Dijk 1992, 2001). La etnografía y los estudios profesionales van indicando, al mismo tiempo, la jerarquización de las élites profesionales, el carácter precario y agresivo de la profesión y la pérdida de prestigio social del periodismo, que, de ser considerado un oficio vital en la vida social, pasa a ser simplemente uno de los menos valorados, si no el menos, conforme avanza el fin del siglo XX (Diezhandino y Nieto, 1995).

Si es verdad que los estudios son cada vez más certeros, cada vez pasan menos al conocimiento de los propios profesionales. Las investigaciones son más complejas, pero también sus expositores son más oscurantistas. Los estudios de diagnóstico son en esos años abiertamente ignorados por los profesionales, que viven una era dorada en el ejercicio profesional, con tiradas millonarias y audiencias inmensas. Se vive la palmaria contradicción de conocer en detalle todos los vicios y degeneraciones del periodismo, y de presenciarlos a diario obteniendo además enorme éxito social. Todo ello no tiene trascendencia alguna en la evolución profesional. Anecdóticamente puede que surjan recomendaciones o decisiones en algunas asociaciones profesionales. Pero el periodismo en masa no solamente no reacciona ante las investigaciones, sino que las ignora abiertamente. En España tenemos claros ejemplos de análisis sobre el sensacionalismo informativo de los años finales del siglo XX, que no solamente no generan decisiones en las asociaciones profesiona- 
les, sino que son rechazados por muchos periodistas bajo el imperativo del profesionalismo salvaje.

\section{EL COMIENZO DEL FIN. EL ADVENIMIENTO DE LA CRISIS TECNOLÓGICA}

El periodismo popular sigue su camino hacia la degeneración absoluta en medios como las revistas y la televisión al comienzo del siglo XXI. Los géneros basados en principios inmorales - cotilleo, morbo, robo de información, violencia informativatriunfan aparentemente durante toda una década en estos sectores. La visibilidad profesional de los periodistas se ve seriamente dañada por estas prácticas.

En esa inconsciente edad dorada del periodismo sensacionalista o protocolario, muchos silenciosos enemigos han entrado por la puerta del cambio tecnológico. El primero de ellos es el advenimiento de Internet, y el cambio inmenso que pone patas arriba el diseño perfectamente aherrojado del control de la información y de las fuentes que los periodistas llevan un siglo trabajándose. La web muta la vida social hacia un modelo de abundancia informativa, no de escasez. Establece una nueva relación entre receptores y medios, dando a los primeros acceso a múltiples fuentes de información en bruto. Los estudios descriptivos del cambio (Manovich 2005, Bozkowski 2006) indican que son múltiples las convulsiones en el viejo modelo periodístico. Cambios estructurales centrales como los que afectan a los derechos de autor, el acceso directo a fuentes políticas, democráticas, académicas, la nueva reversibilidad del proceso informativo, la creación de nuevos medios informativos, la gestión de la información personalizada. El cambio es tan apasionante como rápido. Pero la profesión periodística, secularmente interesada en no cambiar sus rutinas y en defenderse de las evaluaciones, no está preparada para la revolución que llega. El estatismo sigue siendo uno de los rasgos del periodismo de 2010 (Boczkowski, 2013), su incapacidad para adaptarse a las demandas cambiantes de los lectores.

El elemento clave es fundamentalmente la desaparición de las instancias mediadoras que se adivina para muchos sistemas de acción social: la tecnología en red hace posible una democracia directa, el acceso directo a la información, al conocimiento no mediado o no dispensado por élites en exclusiva. Los sistemas de comunicación explosionan hacia múltiples nuevas posibilidades. El periodismo profesional de referencia, completamente volcado hacia las fuentes de poder, se encuentra con que las fuentes sin poder empiezan a ofrecer información por todas partes, sin posible control. El caso WikiLeaks es un ejemplo de las convulsiones que el modelo periodístico sufre, y de cómo los intentos por reconducir la situación al viejo modelo terminan pasando factura (Lovink y Riemens 2008). La opinión pública empieza a desvincularse nuevamente del periodismo profesional, y a articularse sobre nuevos media sociales.

La historia tecnológica de los últimos años muestra la incapacidad de reacción y adaptación por el profesionalismo autodefensivo fosilizado. Los cambios se imponen sin plantear estrategias racionales por parte de las empresas. No se trata de una modernización tecnológica para una profesión activa y alerta, sino de toda una acti- 
tud profesional encastillada en sistemas de control que han perjudicado a la capacidad del periodismo para ser autónomo frente a los cambios sociales, y que ahora quieren seguir conservándolos cuando ya no sirven para nada. El periodismo ha perdido, además, gran parte de la confianza en sus motivaciones éticas por parte de la ciudadanía. Cualquier proclamación de la calidad del trabajo periodístico profesional es sospechosa de ser realmente una autodefensa reaccionaria. La calidad en el manejo de la información ha sido abandonada por demasiados periodistas, como para que ahora sea demandada por todos los lectores.

El periodismo en todos los países desarrollados asiste impotente a una sangría de lectores y de consumidores, que va mermando progresivamente su base de apoyo social y sus ingresos y justificación de ingresos publicitarios. Los nuevos medios sociales, los blogs, las webs, empiezan a ejercer un periodismo desindustrializado, más parecido al periodismo del comienzo de su historia que los medios consolidados. Los medios de masas ya no son masivos, y su principal posición en la vida social cae. No es necesario profundizar mucho en la red para captar cómo los lectores críticos dan sus propias visiones de la tarea informativa en las redes sociales como Twitter o Facebook. Si el periodismo siempre había sido una profesión vulnerable a las críticas, ahora lo será muchísimo más.

\section{EL GOLPE DE GRACIA: LA CRISIS FINANCIERA Y EL FIN DEL PERIO- DISMO DE REFERENCIA}

Con el año 2005 llega la crisis financiera, que produce en el periodismo asentado y asociado con los grandes poderes fácticos una ruptura de los lazos de mutua conveniencia que existían. Este terrible hecho hace que los periódicos que se han alineado con fuentes de poder financiero queden absolutamente en sus manos, abandonados a las nuevas estrategias financieras, los recortes y la visión externalizada y privatizada del oficio. La dependencia ahora no es lucrativa, sino crediticia. Los periódicos no es que se vendan al poder, es que están endeudados con él. Su independencia es ya mera fantasía. Y los lectores pueden ver, desde Internet, las maniobras que la dependencia financiera impone en los medios considerados de élite, obligándoles a cambiar de enfoques, reducir calidad del producto, dar la visión más reaccionaria de la propia situación de cambio social.

La crisis del sistema político, la crisis de confianza en el sistema representativo de los políticos profesionales, es otra catástrofe paralela y diríamos que hermana de la crisis del periodismo. Hemos visto que Lippman, en los años 20, ya diagnosticaba que ambos eran causa y efecto de un mismo proceso. Los mismos procesos de profesionalización salvaje han llevado a ambos oficios a perder su horizonte ético y a vaciarse de valor social. Se trata de dos de las profesiones menos valoradas en las encuestas sociales de 2010. De nuevo se nota la desaparición del valor simbólico de las instancias mediadoras, en una sociedad en la que ya no son necesarias, y en la que además, se han vuelto indeseables, por su corrupción y por su ejercicio de falsa conciencia.

Muchos analistas se preguntan cuál puede ser la salida para una profesión asediada por cinco crisis estructurales, infraestructurales, superestructurales de todo 
tipo. La pérdida de credibilidad social es un largo proceso que comenzó en los años 50. La incapacidad de adaptarse a los cambios se labró durante décadas en una profesión con resistencia al cambio. El acomodo, la renuncia al ejercicio difícil y complejo de una profesión única en la vida social, pasan finalmente factura. La falta de articulación profunda del oficio lo hace incapaz de bandear las dificultades que surgen por todas partes, que jamás pudieran haberse previsto. Pero si el periodismo hubiera conservado su función original de conciencia social ciudadana, habría preservado su núcleo de valor de la vorágine de cambiantes circunstancias externas.

Mi opinión es que una de las razones ocultas de esta crisis es la debilidad de las categorías básicas, de los valores en profundidad, y de la investigación ontológica sobre el periodismo profesional que tanto profesionales como teóricos han aceptado durante décadas. Los periodistas han ejercido un oficio que ha bailado demasiado de ideales y funciones. La academia, los investigadores del mundo profesional, no han avanzado tampoco en espolear a la profesión para que existiera consciencia de los cambios que se estaban dando. Quizás sea tiempo ya de que las Teorías Profesionales empiecen a señalar el camino a seguir, y dejen de indicar el abismo al que el periodismo profesional se dirige.

\section{PERO ¿QUÉ ES REALMENTE UNA NOTICIA? UN CONCEPTO INÉDITO DEL PERIODISMO}

Durante décadas me he preguntado realmente qué son las noticias, obligada a estudiar el periodismo profesional para poder enseñar lo que es. Las definiciones descriptivas de las noticias son tremendamente anodinas. Las teorías profesionales, con su eje crítico, dieron cierta vitalidad a la reflexión sobre qué es una noticia pero siempre, como indico, desde un punto de vista crítico poco constructivo: como la célebre definición de Herbert Gans en los años 70 :"una noticia es el ejercicio de poder sobre la interpretación de la realidad". (Gans, 1960 ). La aguda definición del norteamericano no hacía sino constatar un fracaso profesional. Seguíamos sin saber qué era una noticia.

Aún siendo interesantes, las definiciones de noticia que conozco han dejado completamente a oscuras lo que realmente debe ser una noticia. Es un ejemplo claro de cómo el campo categorial del periodismo, que es vital para entender la base humana del oficio, está todavía por analizar.

La definición más simple es en muchas ocasiones un núcleo de significado muy profundo. Cuando pensamos en la noticia, la definimos como una novedad. Y esta simple definición (una noticia es una novedad en la vida social) tiene, como estudiara Robert Ezra Park a comienzos de nuestra época, un profundo valor. (vid aquí mismo su artículo y en Mc Chesney 2011)

Las noticias no son relatos de novedades, sino novedades cognitivas en sí. Cuando recibimos una noticia, se produce una complicada actividad en nuestra mente, que adopta y asimila algo realmente desconocido hasta ese instante. Los periodistas que redactan auténticas noticias hacen aprender a sus lectores cosas nue- 
vas, obligándoles a avanzar en el conocimiento, y con ello, movilizándolos en su vida social.

Para mí, una noticia es una avanzadila en la evolución social: es un producto realizado por un informador, capaz de tirar del conocimiento público hasta hacerlo progresar y adelantar puestos en su evolución humana. Esta función es la que da nobleza y altura a las noticias, pues los periodistas fuerzan a la sociedad a crecer y elevarse, educan a los lectores, presionan para el avance en la vida social.

En su aspecto más profundo y cierto, el periodismo es una profesión educativa. Cuando los periodistas aglutinan en torno de los medios a la mayoría social, enseñándola a adoptar innovaciones e insertándola en el dinamismo natural de la existencia, están haciendo algo mágico, increíble, único. Consiguen mover el gigantesco y lento mecanismo de la educación ciudadana, y activarlo para generar un mundo nuevo. Parece poca cosa una noticia, pero si las noticias son novedades, su papel en la vida social es verdaderamente excepcional.

El periodismo tiene una función excepcional en la activación del conocimiento como base de la actividad social. Los públicos informados se convierten en ciudadanía responsable, activa, capaz de tomar decisiones y de actuar cuando es preciso contra el poder despótico, la corrupción o la propaganda, como afirmaba Lippman en 1920. Siendo una profesión educativa, sin embargo el periodismo debe convertirse en un sistema dinámico y cambiante de adquisición de información, en el que se aprenda sin tener conciencia de estar aprendiendo. El sistema educativo del periodismo está liberado de las constricciones académicas. Por eso se diferencia de la escuela o de la universidad. Y cumple un papel social en espacios diversos a los de la universidad o la escuela.

Todos sabemos que lo que se ha realizado como noticias, por miles y miles de periodistas, durante siglos, no ha sido esto precisamente. El cuadro que hemos descrito del periodismo lo aleja definitivamente de las auténticas noticias, es decir, de esos misteriosos aceleradores del desarrollo humano.

Parece que una de las exigencias de la situación actual es la refundación del ciclo moral del periodismo, la política y otras profesiones de mediación simbólica. Una vez que el oficio periodístico no puede ya autodefenderse de los cambios y ha perdido sus mecanismos de permanencia y estabilidad, se impone recuperar la función social de abanderados del cambio y el progreso. El periodismo ciudadano, despojado del poder masivo, del poder de negociar con el poder, obedece a este nuevo regeneracionismo profesional que es sin duda una de las demandas sociales más claras sobre la profesión.

El periodismo ha estado siempre volcado hacia el sistema de las fuentes de información, de cuya escasez obtenía los privilegios de difusión. El actual esquema comunicativo de nuestra sociedad ha cambiado radicalmente. El dinamismo informativo está ahora en los receptores: ellos son ahora la noticia. Los públicos están experimentando tal mutación cognitiva que se convierten en el nuevo foco de interés social. Las redes sociales son el símbolo del nuevo papel activo, dinámico, abundante en información, que tienen los habituales receptores del periodismo. Como ahora se dice, ellos son la información, a ellos llegan las noticias sin buscarlas. Y ello exige que los periodistas se vuelvan a los públicos y que registren este fabulo- 
so cambio del esquema comunicativo social. Que comiencen a notar que tienen ante sí el desafío de informar directamente sobre el magma social en fusión que está generando una nueva era. Es decir, que empiecen a ejercer el periodismo como nunca se ha ejercido.

El activismo no es un desconocido para la conformación del periodismo como profesión. Robert Ezra Park afirmaba que las noticias obligaban a las sociedades a actuar, a participar activamente en la vida global. El activismo ciudadano que hoy es vital necesita del periodismo como factor de publicitación, y necesita de un periodismo alineado y comprometido con él, como garante de la preservación de las libertades sociales hoy en peligro de represión y recesión.

El periodismo no lucrativo tampoco es un fantasma en el mundo profesional. El periodismo que nació con el siglo XX era una profesión voluntaria, no lucrativa, valiente, aventurada. En ella lo último era el beneficio, lo primero era cumplir un deber. La profesión periodística tenía mucho de gremial, bohemia, heróica. Algo de eso vemos en los nuevos blogs, en los periodistas que realizan Streamings en las manifestaciones, en los nuevos tipos de informadores que en muchos casos ejercen esta actividad de manera voluntaria, gratuita, como causas personales y compromisos individuales . Todas aquellas imágenes ideales del periodismo se han convertido en necesidades hoy que deben asumirse por los profesionales para sobrevivir a la convulsión autodestructiva que está acabando con los periódicos. Si no es así, el periodismo continuará sangrando por su principal herida mortal: la de la pérdida de su utilidad social.

\section{BIBLIOGRAFÍA}

Boczkowski Pablo (2006) Digitalizar Las Noticias. Innovación en los diarios online. Buenos Aires, Manantial

BoczKowsKi, P. (2010, 2013): "Preferencias Divergentes De Los Lectores Y De Los Periodistas En Las Noticias On-Line".Publicado originalmente en la Revista Acm | November 2010 | vol. 53 | no. 11 . Traducido en revista CIC. 2013, 18.

BreED, Warren (1955):, «Social Control in the Newsroom: A Functional Analysis» Social

Forces, Vol. 33, No. 4 (May, 1955), pp. 326-335 Published by: University of North

Carolina Press

Diezhandino Nieto, Pilar, et al (1995). La élite de los periodistas. Bilbao, UPV.

Elliott Philip (1975) Sociología de las profesiones. Madrid, Tecnos.

FishMAN Mark (1983) La fabricación de la noticia. Chile, Tres Tiempos.

Galtung, Johan, Ruge, Mari. (1965) "The structure of foreign news: The presentation of the

Congo, Cuba and Cyprus crises in four foreign newspapers". Journal of International

Peace Research, vol. 1, pp. 64-90.

Gans, Herbert (1960) Deciding What's News: A Study of CBS Evening News, NBC Nightly

News, Newsweek, and Time. Northwestern, Northwestern Univ. Press

Glasgow Media Group, Bad News. 1976.London, Routledge.

Goldstein, Tom, The News at Any Cost.(1982) New York, Simmon and Schuster.

IMBERT, Gerard y VidAl BENEYTo, José (1986), El Pais o la referencia dominante. Barcelona, Gedisa. 
Lovink, Gertz y RiEmens, Patrick (2008 2011), "Doce tesis sobre WikiLeaks", Cuadernos de Información y Comunicación, 16, pp.139-147.

Manning White, David, White, David Manning (1950). “The "gate keeper": A case study in the selection of news ". Journalism Quarterly 27: 383-391.

MC CHESNEY, Robert W (2011)., "That was now and this is then: Walter Lippman and the crisis of journalism", en McChesney, R., y Pickard, V. (2011) Will the last reporter turno ut the lights when he comes out. New York, The New Press. Trad. en revista CIC, 18, 2013.

ManOvich, Lev(2005), El lenguaje de los nuevos medios de comunicación. Madrid, Cátedra.

Paletz, David and Robert M. Entman (1980) "Presidents, Power and the Press."Presidential Studies Quarterly, 10, 416-26.

PARK, Robert Ezra (1941, 2013), "La moral y las noticias", Cuadernos de Información y Comunicación, 18.

SAnTORO, Guillermo (2004) Tecnicas de Investigación. México, FCE.

Tunstall, Jeremy, (1976) Journalists at Work: Specialist Correspondents, Their News Organizations, News-sources and Competitor-colleagues London, Harmondsworth: Penguin.

Noelle Neumann, E. (1995), La espiral del silencio. Opinión pública, nuestra piel social. Barcelona, Paidós.

Tuchman, Gaye (1986) La producción de la noticia. Barcelona, Gustavo Gili.

VAN DiJK, 1992, 2001 La noticia como discurso. Barcelona, Paidós.

VAlbuena, Felicísimo, Teoría general de la Información. Madrid, Noésis, 1998.

Weil Simone, (1931, 1986). La condición obrera, Paris, Gallimard.

\title{
RESUMEN
}

La autora conecta los diferentes períodos de desarrollo profesional del periodismo con las consecutives crisis de las organizaciones mediáticas y con las investigaciones académicas sobre esta profesión a lo largo de su historia, mostrando el divorcio academic existente entre la profesión y su análisis academic.

Palabras clave: crisis del periodismo, teorías profesionales de las noticias, períodos de desarrollo del periodismo, ética.

\begin{abstract}
The author connects the different periods of professional development in journalism of developed societies with the consecutive crisis of the media, and with the research of academical theorists about news making organisations, establishing a link between the problems appeared in journalism along its history, the surfeit of crises, and the academical divorce from the profession.
\end{abstract}

Keywords: journalism in crisis, professional theories of the news, periods of developments of professional journalism, ethics.

\section{RÉSUMÉ}

Cet article met en connexion les divers étapes de développement du journalisme professional avec les crises que les organisations informatives ont subi pendant leur histoire, et aussi avec la connaissance académique du contexte professional, en montrant le divorce existant entre le journalisme actif et la théorie académique.

Mots clé: crise du journalisme, théories professionnelles des organisations informatives, phases de développement du journalisme professional, étique. 